\title{
Baseline Assessment of Public Health Surveillance in the Kingdom of Swaziland
}

\author{
Harriet Nuwagaba-Biribonwoha ${ }^{* 2}$, Nhlanhla Nhlabatsi ${ }^{3}$, William MacWright ${ }^{1}$, \\ Vusie Lokotfwako ${ }^{3}$, Tony A. Trong ${ }^{4}$, Paige Ryland ${ }^{1}$, Affan Shaikh ${ }^{1}$, Joy Sylvester ${ }^{1}$ and \\ Scott J. McNabb'
}

${ }^{1}$ Public Health Practice, LLC, Belmont, MA, USA; ${ }^{2}$ CAP at Columbia University, New York, NY, USA; ${ }^{3}$ Epidemiology and Disease Control Unit, Swaziland Ministry of Health, Mbabane, Swaziland; ${ }^{4}$ U.S. Centers for Disease Control and Prevention, Atlanta, GA, USA

\section{Objective}

To assess essential support functions for Integrated Disease Surveillance and Response(IDSR) in the Kingdom of Swaziland and make recommendations for a national IDSR Roadmap.

\section{Introduction}

Implementation of the IDSR framework for fulfillment of the International Health Regulations (2005) ([IHR 2005]) has been challenging in Swaziland due to distribution of IDSR functions across units within the Strategic Information Department (SID) and other external departments within the Ministry of Health. We conducted a qualitative assessment and a Strength, Weaknesses, Opportunities and Threats (SWOT) analysis of current public health surveillance (PHS) support structures to inform implementation of IDSR.

\section{Methods}

Key informant interviews, focus group discussions, and a desk review were performed. Participants were personnel at essential units, departments and programs at the national level as well as at health facilities and clinics at regional and local levels. Transcripts were coded into SWOT matrices using MAXQDA for each building block of PHS: structures, workforce, resources, processes (detect, report, assess/analyze, respond, feedback), and informatics.

\section{Results}

Selected Strengths included existence of immediate notifiable disease reporting through the Epidemic and Pandemic Response unit (EPR) and reporting of summary health facility data to the Health Management Information System (HMIS) unit and laboratory network. Weaknesses included lack of clear roles and responsibilities for IDSR among SID units, limited coordination between SID units, lack of data sharing, lack of Standard Operating Procedures (SOPs), uncoordinated case investigations and response, minimal analysis conducted for public health surveillance and limited feedback for reporters.

Identified opportunities were political will for establishing of roles and responsibilities and mechanisms for coordination and data sharing. Threats were limited data access, limited funding for feedback, lack of analysis for IDSR and paper-based reporting

\section{Conclusions}

Currently there is limited use of surveillance data for decision making due to lack of coordination. Findings were presented at a dissemination meeting to representatives of relevant departments, and there was consensus on the need to clearly define the role and responsibilities of different programs for IDSR. In March 2016, a consensus meeting was held to designate roles and responsibilities for IDSR, a direct result of this assessment. Additional resources and funding is needed to support these highly important initiatives to ensure the safety and health security of the Swazi nation.

\section{Keywords}

Africa; Public Health Surveillance; IDSR; Public Health Surveillance; Data Sharing

\section{Acknowledgments}

This work is supported by the President's Emergency Plan for AIDS Relief (PEPFAR) through the Centers for Disease Control and Prevention under the terms of Cooperative Agreement Number 1U2GGH001271. Its contents are solely the responsibility of the authors and do not necessarily represent the official views of PEPFAR or the Centers for Disease Control and Prevention.

*Harriet Nuwagaba-Biribonwoha

E-mail: hn2158@cumc.columbia.edu 